\title{
Photocatalytic Activity Indicator Inks for Probing a Wide Range of Surfaces
}

\author{
Suresh Pillai \\ Technological University Dublin, suresh.pillai@tudublin.ie \\ Andrew Mills \\ Queen's University - Belfast \\ James Hepburn \\ Queen's University - Belfast
}

See next page for additional authors

Follow this and additional works at: https://arrow.tudublin.ie/ehsiart

Part of the Physical Sciences and Mathematics Commons

\section{Recommended Citation}

Pillai, S. et al. (2014) Photocatalytic Activity Indicator Inks for Probing a Wide Range of Surfaces. Journal of Photochemistry and Photobiology A: Chemistryvol. 290 (2014) pp63-71 doi:10.1016/ j.jphotochem.2014.06.007

This Article is brought to you for free and open access by the ESHI Publications at ARROW@TU Dublin. It has been accepted for inclusion in Articles by an authorized administrator of ARROW@TU Dublin. For more information, please contact arrow.admin@tudublin.ie, aisling.coyne@tudublin.ie,gerard.connolly@tudublin.ie.

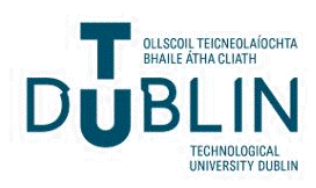




\section{Authors}

Suresh Pillai, Andrew Mills, James Hepburn, David Hazafy, Christopher O'Rourke, Nathan Wells, Josef Krysa, Michal Baudys, Martin Zlamal, Hana Bartkova, Claire E. Hill, Kim R. Winn, Morten E. Simonsen, Erik G. Sogaard, Swagata Banerjee, and Rachel Fagan 
Cite this as Journal of Photochemistry and Photobiology A: Chemistry 290 (2014) 63-71

Article link: http://www.sciencedirect.com/science/article/pii/S1010603014002597

\title{
Photocatalytic Activity Indicator Inks for Probing a Wide Range of Surfaces
}

\begin{abstract}
Andrew Mills*, James Hepburn, David Hazafy, Christopher O'Rourke and Nathan Wells

School of Chemistry \& Chemical Engineering, Queen's University Belfast, Northern Ireland, $U K$
\end{abstract}

\section{Josef Krysa, Michal Baudys, Martin Zlamal and Hana Bartkova}

Department of Inorganic Technology, Institute of Chemical Technology Prague, Technická 5, Prague 166 28, Czech Republic

\section{Claire E. Hill, Kim R. Winn}

Cristal Pigment UK Ltd., Laporte Road, Stallingborough, Grimsby, North East Lincolnshire, $U K$.

Morten E. Simonsen and Erik G. Søgaard, Section of Chemical Engineering, Department of Chemistry and Biotechnology, Aalborg University, Denmark

\author{
Swagata Banerjee, ${ }^{1}$ Rachel Fagan ${ }^{1,2}$ and Suresh C. Pillai ${ }^{1,3}$ \\ ${ }^{1}$ Centre for Research in Engineering Surface Technology (CREST), FOCAS Institute, Dublin \\ Institute of Technology, Kevin St, Dublin 8, Ireland ${ }^{2}$ School of Chemical and Pharmaceutical \\ Sciences, Dublin Institute of Technology, Kevin St., Dublin 8, Ireland ${ }^{3}$ Department of \\ Environmental Science, Institute of Technology Sligo, Sligo, Ireland
}

Key words: photocatalysis, inks, standards, Resazurin, Acid violet 7, Basic blue 66 Journal of Photochemistry and Photobiology A: Chemistry 290 (2014) 63-71 


\section{Abstract}

Three photocatalyst inks based on the redox dyes, Resazurin (Rz), Basic Blue 66 (BB66) and Acid Violet 7 (AV7, are used to assess the photocatalytic activities of a variety of different materials, such as commercial paint, tiles and glass and laboratory made samples of sol-gel coated glass and paint, which collectively exhibit a wide range of activities that cannot currently be probed by any one of the existing ISO tests. Unlike the ISO tests, the ink tests are fast (typically $<10 \mathrm{~min}$ ), simple to employ and inexpensive. Previous work indicates that the $\mathrm{Rz}$ ink test at least correlates linearly with other photocatalytic tests such as the photomineralisation of stearic acid. The average time to bleach $90 \%$ of the key RGB colour component of the ink (red for Rz and BB66 inks) and green for AV7 ink) is determined, $t t b(90)$, for eight samples of each of the different materials tested. Five laboratories conducted the tests and the results revealed an average repeatability and reproducibility of: ca. $11 \%$ and ca $21 \%$, respectively, which compare well with those reported for the current ISO tests. Additional work on commercial self-cleaning glass using an Rz ink showed that the change in the red component of the RGB image of the ink correlated linearly with that of the change of absorbance (at $608 \mathrm{~nm}$ ) (as measured using UV/Vis spectroscopy) and the change in the $\mathrm{a}^{*}$ component of the Lab colour analysis of the ink, as measured using diffuse reflectance spectroscopy. As a consequence, all three methods generate the same $t t b(90)$. The advantages of the RGB digital image analysis method are discussed briefly.

\section{Introduction}

A number of different ISO tests have been developed to date to assess the activities of photocatalytic surfaces, however, most, if not all, are only able to probe a limited range of activities $^{1}$. For example, the methylene blue (MB) test (ISO 10678:2010) is appropriate for moderately active materials, like most examples of commercial self-cleaning/easy clean glass, but is of little use for assessing the high activity, materials, like thick sol-gel titania films on glass, or photocatalyst paper and paints, due to the low diffusion coefficient of MB in solution ${ }^{1,2}$. Nor is the MB test effective in probing the efficacy of low activity materials, such as most commercial photocatalytic tiles, due to the low, but measurable UV photoinstability of $\mathrm{MB}^{2}$. The ISO air purification tests, such as the ones based on the removal of: NOx (ISO 22197-1), acetaldehyde (ISO22197-2) or toluene (ISO 22197-3:2011), are very good for assessing high (but not very high) activity, flat, non-porous materials ${ }^{1}$, but poor for exploring the activities of moderately active flat, non-porous materials, such as self-cleaning glass, due to the high flow rates and low pollutant concentrations used ${ }^{1}$. In addition to the 
above, all of the ISO tests are slow (typically of 3-5 h duration) and require expensive analytical equipment and technical support ${ }^{1}$. Clearly, it would be desirable, therefore, if a test method could be identified which was able to probe simply, rapidly and cheaply, the wide range of activities exhibited by the many different photocatalytic surfaces there are available currently, such as those found in commercial tiles, paints and glass.

In a recent paper we reported on a simple, inexpensive method for the rapid testing of the photocatalyst activity of self-cleaning surfaces of moderate activity, such as commercial selfcleaning glass ${ }^{3}$. In that paper, a photocatalyst activity indicator ink was used, comprising a dye, Resazurin (Rz), a sacrificial electron donor (i.e. SED, e.g. glycerol) and a polymer, hydroxyl ethyl cellulose (HEC) to encapsulate the dye and SED, when the ink had dried. This ink functions via a photo-reductive mechanism summarised by reactions (1) - (3), where SC is the underlying semiconductor photocatalyst film onto which the ink film is placed and allowed to dry, $D_{o x}$ is the dye in its original, and usually highly coloured, (oxidised) form (blue in the case of $\mathrm{Rz}$ ) and $D_{\text {red }}$ is the reduced, and differently coloured, form of the dye (pink in the case of Rz, but colourless for the other dyes used in this work).

$$
\begin{aligned}
\mathrm{SC} & \stackrel{h v>E_{b g}}{\longrightarrow} \mathrm{SC}^{*}\left(\mathrm{~h}^{+}, \mathrm{e}^{-}\right) \\
\mathrm{SED}+\mathrm{SC}^{*}\left(\mathrm{~h}^{+}, \mathrm{e}^{-}\right) & \rightarrow \mathrm{SED}_{\mathrm{ox}}+\mathrm{SC}\left(\mathrm{e}^{-}\right) \\
D_{o x} & \rightarrow D_{\text {red }}
\end{aligned}
$$

$E_{b g}$ is the band gap energy of the semiconductor, which is almost always titania, often in its anatase form (and so $E_{b g}=3,0 \mathrm{eV}$ ), and $\mathrm{SED}$ and $\mathrm{SED}_{\mathrm{ox}}$ are glycerol and glyceric acid/glyceraldehyde, respectively. Previous work reveals that the rate of the above, Rz inkbased, photocatalyst driven process, is directly correlated with the much slower destruction of a film of stearic acid (SA) on photocatalyst films on glass ${ }^{4}$.

Following on from the above work, in this paper this same simple method has been expanded, and now uses not only the Rz ink but also two other different photocatalyst activity inks, so as to be able to assess the activity of a number of different flat, smooth, largely nonporous photocatalytic materials, such as glass, paint and tiles, which collectively exhibit a wide range of photocatalytic activities. 


\section{Experimental}

\section{Chemicals and Materials}

All chemicals were purchased from Sigma-Aldrich and, unless otherwise stated, were used as received. The water used to produce all inks was double distilled and deionised. The commercial self-cleaning glass used to test the ink was BioClean ${ }^{\circledR}$ supplied by Saint-Gobain, the commercial photocatalytic tiles were from Deutsche Steinzeug and the commercial, fast, photocatalytic paint (StoClimasan Color) was purchased from Sto Ltd. The 'fast' glass photocatalyst samples were prepared, by ICT, using a sol-gel technique according to a previously reported method ${ }^{5}$ and the medium activity paint was prepared by QUB, by mixing $5 \%$ (by volume) of a highly active photocatalytic paint based on PC50 anatase titania, with one that exhibits no activity, with both paints being provided by Cristal. Coated samples of these medium and high activity paints were made using a No. 4 K-bar - a wire-wound rod (delivering a wet coating of 40 microns) to draw-down a layer of paint to cover a glass microscope slide, $26 \mathrm{~mm} \times 76 \mathrm{~mm}$. All paint-coated samples were then left to dry in the dark under ambient conditions for 30 minutes and used without any further treatment.

Preparation of the photocatalyst medium activity indicator ink: the Resazurin ink (Rz)

The Rz ink was made by dissolving 0.15 g HEC (CAS No.: 9004-62-0; viscosity: 145 mPa s for $1 \mathrm{wt} \%$ solution in water) into $9.85 \mathrm{~g}$ high purity (conductivity $\leq 2 \mu \mathrm{S} \mathrm{cm}{ }^{-1}$ ) water to give a solution of $1.5 \mathrm{wt} \% .1 \mathrm{~g}$ of glycerol was then added to this polymer solution, followed by 10 mg of Rz, (CAS No.: 62758-13-8; purity 75\%) and $20 \mathrm{mg}$ of the surfactant, polysorbate 20 (CAS No.: 9005-64-5). The ink was stirred with a magnetic stirrer for a minimum of 8 hours and then stored in a fridge. The ink was used within 2 weeks of its preparation but, each time, before its application, it was removed from the fridge and stirred on a magnetic stirrer at room temperature for 1 hour.

Preparation of the photocatalyst low activity indicator ink: the Basic Blue 66 ink (BB66) The procedure for making the BB66 ink was identical to that for the Rz ink, except $50 \mathrm{mg}$ of BB66 (CAS No.: 94233-04-2; purity 20\%) were used in place of the Rz.

Preparation of the photocatalyst high activity indicator ink: the Acid Violet 7 ink (AV7)

The procedure for making the AV7 ink was identical to that for the Rz ink, except $25 \mathrm{mg}$ of AV7 (CAS No.: 4321-69-1; purity: 40\%) were used in place of the Rz. 
The structures of the three redox dyes, i.e. Rz, BB66 and AV7 are illustrated in figure 1.
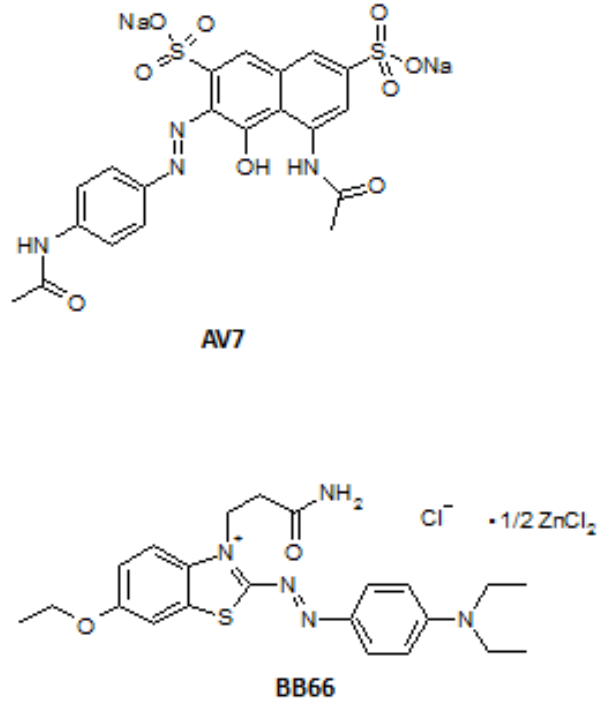
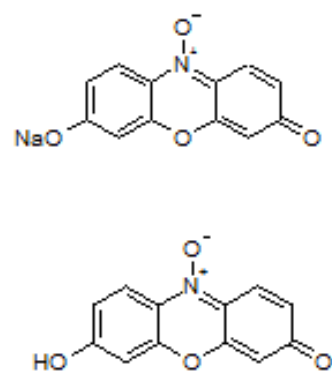

$\mathrm{Rz}$

Figure 1: Structures of the three redox dyes (Resazurin (Rz), Basic Blue 66 (BB66) and Acid Violet 7 (AV7) used in this work

\section{Sample size, number and pre-treatment}

The materials under test were cut into ca. $25 \mathrm{~mm} \times 25 \mathrm{~mm}$ squares and 8 samples used for each test. The ink tests are ideal for testing samples in almost any condition although, usually, this will be preferably in a pristine (i.e. usually very clean) form. In order to achieve the latter state, a cleaning protocol will usually have been performed, such as: a thorough wiping with a water-soaked cloth (to remove any dirt) followed by 15-24 h irradiation with UVA light, as is recommended by most of the existing ISO photocatalyst test methods ${ }^{1}$. However, the ink tests can also be used to probe the activities of samples in a variety of different states, such as: contaminated, aged or weathered. As a consequence, in order to assess correctly the activities associated with such samples, of what is otherwise the same material, little or no pre-treatment is recommended to form part of the test per se, although details of the sample, its source and any pre-conditioning applied to the sample before the test, should form part of any report made by the tester on the photocatalytic activity of the sample of the material tested. Thus, in this work, most of the materials were cleaned only by wiping lightly with a water-soaked, silicone-free, tissue and then allowed to dry for 60 minutes in the dark under ambient conditions. In the case of the paint samples, however, 
which had surfaces that were more easily damaged than those of the tile and glass samples, no pre-treatment was used.

\section{Coating the sample with the ink}

In order to coat a ca. $25 \times 25 \mathrm{~mm}$ sample with a photocatalyst activity indicator ink, it was secured to an impression bed (i.e. a clipboard) and a line of ink ca. $2.5 \mathrm{~cm}$ long deposited along the length of the top of the sample, using a pipette. The typical amount of ink delivered in this way was ca. $65 \mu \mathrm{L}$. A wire wound rod (K-bar No. 3) was then used to spread/coat the ink onto the sample by drawing the bar down from the top of the sample (where the line of ink was) to the bottom, using sufficient pressure on the bar to ensure the spiral wire remains in contact with the sample throughout the drawdown process, but not so much that the K-bar is bowed during the drawdown process, which produces an uneven coating. This process was then repeated for the other seven samples of the material under test and the whole coating procedure should take less than $15 \mathrm{~min}$. Upon coating the last sample of the material, all the ink-coated samples were then left for 60 mins in the dark, but under otherwise under ambient conditions, before being assessed for photocatalytic activity.

\section{Instrumentation}

All irradiations were conducted using a Blak-Ray ${ }^{\circledR}$ XX-15 lamp and exposure stand purchased from Cole-Parmer. The bulbs used in the lamp were $15 \mathrm{~W}$ Blacklight tubes (Eiko) with $\lambda_{\max }$ emission of $352 \mathrm{~nm}$. The sample tray was set so as to irradiate the samples under test with a UV-A irradiance of $\sim 2 \mathrm{~mW} \mathrm{~cm}^{-2}$, as measured using UVX Digital Ultraviolet Intensity Meter, with a UVX-36 sensor head for UV-A light (both from Cole-Parmer). Typically, the gradual change in colour exhibited by a photocatalyst activity indicator ink coating on an active sample under test was recorded as a function of UVA irradiation time, $t$, via digital photography, using an Ion CopyCat handheld document scanner, which has rollers which ensure a small (3 mm) gap between sample and scanner is maintained and so a consistent image was obtained. Note: for most laboratory-based studies a table scanner can be used instead ${ }^{3}$.

\section{Digital Image Analysis (for semi-qualitative analysis test only)}

For each digital image of an ink-coated sample under test, recorded using the scanner after irradiation time, $t$, commonly available software was used to determine the average individual RGB values, i.e. $R G B(\text { red })_{t}, R G B(\text { green })_{t}$, and $R G B(\text { blue })_{t}$, of the central $1 / 9^{\text {th }}$ (ca. $100 \times 100$ 
pixels) part of the image of the scanned $25 \times 25 \mathrm{~mm}^{2}$ sample. The digital image must have a minimum resolution of $300 \mathrm{dpi}$. ImageJ ${ }^{7}$, is an example of free software for this purpose, but there are numerous others, including Adobe Photoshop®. Using the $R G B(\text { red })_{t}$, $R G B$ (green), and $R G B\left(\right.$ blue) $t$ data thus obtained, a value for $R_{t}$, i.e. the normalised red (when using Rz and BB66 inks) or green (when using AV7 ink) component value, of the RGB digital image of the ink film on the photocatalyst material under test at time $t$ after irradiation, was then calculated, since:

$$
\left.R_{t}=R G B(\text { red })_{t} /(\text { RGB(red })_{t}+R G B(\text { green })_{t}+R G B(\text { blue })_{t}\right)
$$

for Rz and BB66 inks, or

$$
\left.R_{t}=R G B(\text { green })_{t} /(\text { RGB(red })_{t}+R G B(\text { green })_{t}+R G B(\text { blue })_{t}\right)
$$

for the AV7 ink. As a consequence, whatever the ink used, every sample of a material tested in this way, yielded a series of $R_{t}$ versus irradiation time, $t$, values, such as illustrated in figure 2 and from this data, and plot, the values of other key parameters were determined, including: the maximum and minimum values of $R_{t}$, i.e. $R(\max )$ and $R(\min )$, from which a value for $\Delta R$ (total), the overall change in $R_{t}$, was calculated, since:

$$
R(\text { total })=R(\max )-R(\min )
$$

This in turn allowed the calculation of $R_{t}(90)$, the value of $R_{t}$ when $90 \%$ of the overall change in $R_{t}$ (i.e. $90 \%$ change in the key colour parameter) had occurred, since:

$$
R_{t}(90)=0.9 \Delta R(\text { total })+R(\min )
$$

$R_{t}(90)$ is associated with a unique irradiation time, $t t b(90)$, i.e. the irradiation time taken for the ink to undergo $90 \%$ of its overall colour change. In this work a value for $t t b(90)$ was estimated simply from the plot of $R_{t}$, vs $t$ and using a straight line equation which joins the two data points that straddle the value of $R_{t}(90)$, as illustrated in figure 1 . Alternatively, the whole $R_{t}$, vs $t$ data set for any one sample can be curve fitted to an appropriate polynomial, such as: $R_{t}=a+b t+c t^{2}+d t^{3}+e t^{4}$, and a value for $t t b(90)$ determined by solving this equation for $t$, given the known optimised fit values (in this case of: $a, b, c, d$ and $e$ ) and that at $t=t t b(90), R_{t}=R_{t}(90)$. 


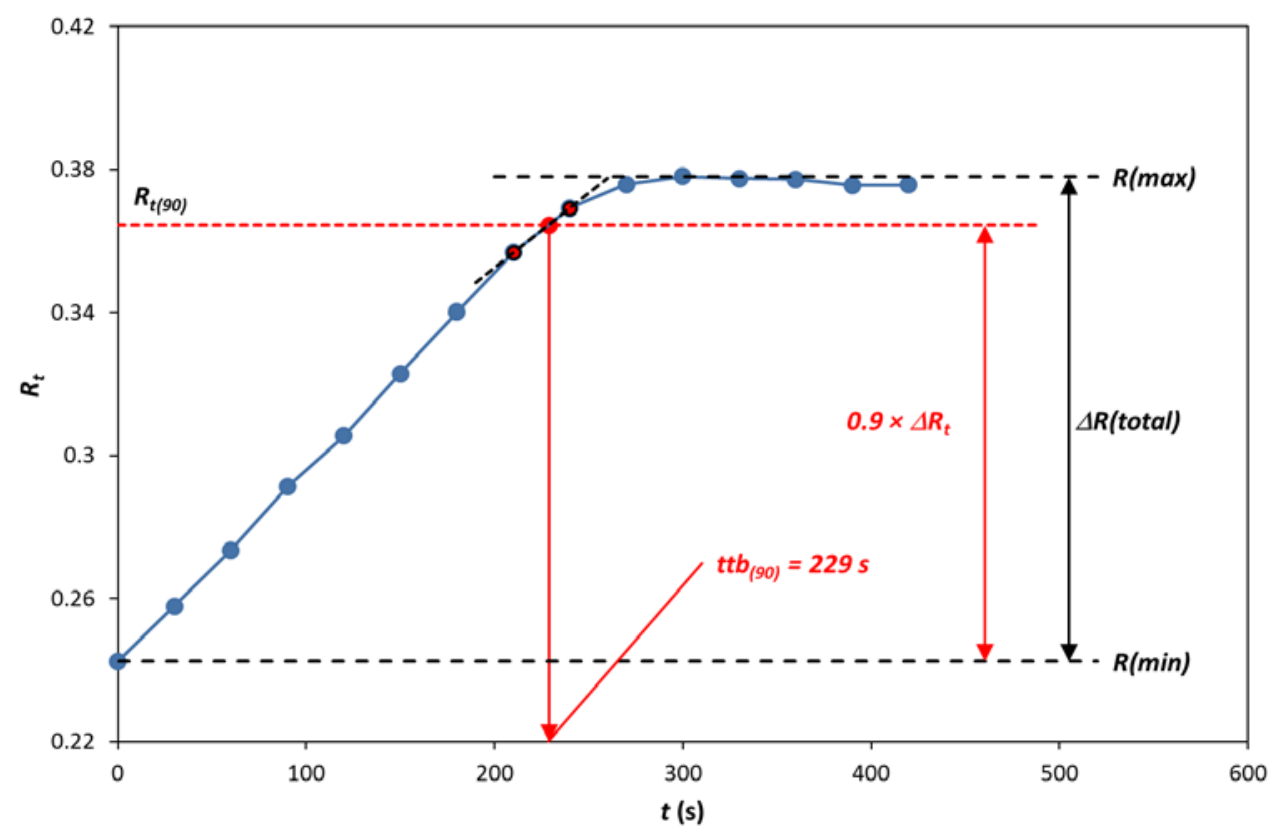

Figure 2: Illustrative plot of $R_{t} v s t$ for an Rz ink on a photocatalytic sample, highlighting the simple least-squares method of determining $t t b(90)$.

\section{Results and Discussion}

Five institutions, namely: QUB, ICT, Cristal, Aalborg and DIT, were involved in conducting a series of round robin (RR) tests of five different photocatalytic materials that each exhibit different, and in some cases very different, activities compared to each other. However, before running the RR tests, each material was first tested at QUB to identify which, of the three photocatalyst activity indicator inks, was appropriate for use with the material under test in the RR tests. All the materials were first tested using the $\mathrm{Rz}$ ink and the results of this work revealed that the commercial self-cleaning glass and medium activity paint changed the colour of the $\mathrm{Rz}$ ink on a reasonable timescale (i.e. exhibited $t t b(90)$ values which lay between 4-6 $\mathrm{min}$ ) - and so were suitable for the RR trials of these materials - but that the fast glass, and fast commercial paint samples went too fast (i.e. $t \mathrm{tb}(90)<90$ s!) and the commercial tiles went much too slow (i.e. $t t b(90)>45$ min!). Previous work carried out by the QUB group had identified two azo dyes, BB66 and AV7, which, when used in a similar ink formulation to that of an Rz ink (see Experimental section), photobleached, respectively, at much faster and slower rates than the $\mathrm{Rz}$ ink $^{7}$. As a consequence, the BB66 ink was used in the RR trials to assess the activity of the low activity commercial tiles and the AV7 ink was used in the RR trials to assess the different, high activities of the fast glass and fast 
commercial photocatalytic paint samples. The results of this work are summarised in the following sections.

\section{The Rz ink test}

Our previous paper showed that the Rz ink test was very well suited for measuring the photocatalytic activity of commercial self-cleaning glass - which can be classed as a material of medium activity ${ }^{3}$. In the latter work, a measure of the activity was taken as the inverse of the time taken to bleach the red component of the $\mathrm{Rz}$ in, i.e. $t t b$, which was the time associated with the point of intersection of two straight line sections drawn from opposite parts of the same $R_{t}$ vs $t$ profile $^{3}$. However, this simple graphical method suffers a little in terms of rigour due to the subjective nature of the choice of the exact positions of the two opposing lines. This negative feature became more apparent when inks other than Rz were used and so, in this work, a different, a more objective method was effected in which a new parameter, $t t b(90)$, was calculated instead, as described earlier, for each of the recorded $R_{t}$ vs $t$ profiles (see Figure 2). Thus, for the Rz ink on commercial self-cleaning glass RR data set generated by Cristal, the subsequent calculated $R_{t}$ vs $t$ profiles for the eight samples are illustrated in figure 3 , on which are also plotted the calculated $t t b(90)$ values (red squares).

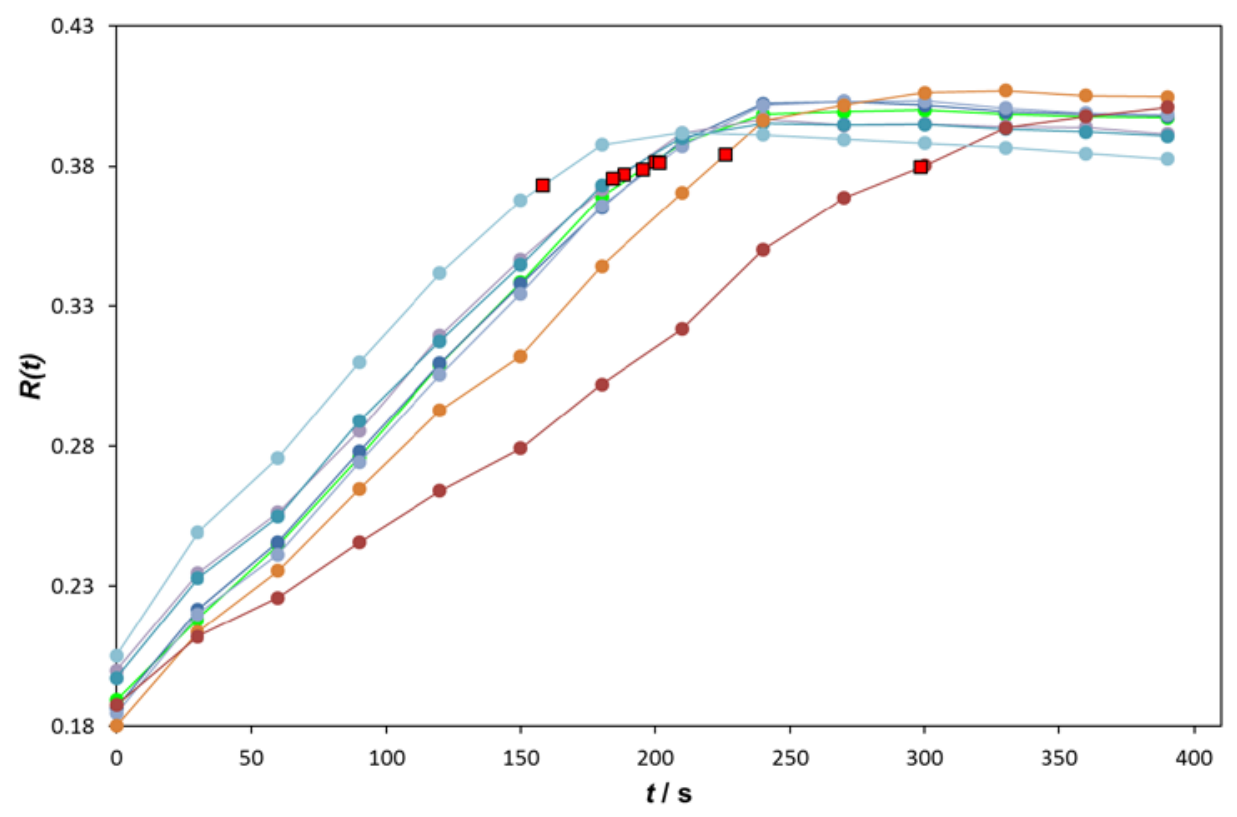

Figure 3: Plots of $R_{t}$ vs $t$ profiles for an Rz ink on 8 samples of commercial glass as reported by Cristal as part of the RR tests. The solid red box points identify the calculated $t t b(90)$ values for each of the curves. 
As can be seen from the data in Figure 3, although most of the $R_{t}$ vs $t$ profiles are very similar, it is not unusual to find at least one profile, usually generated at the beginning of the ink coating process for the 8 samples, which is noticeably different from the rest and so likely to generate a very different $t t b(90)$ value compared to those of the other samples. In order to identify and remove such statistically significant outliers, a modified Z-score, Z(mod), was calculated for each $t t b(90)$ value, where:

$$
Z(\text { mod })=0.6754(t t b(90)-\text { median }(t t b(90))) / M A D
$$

and $M A D=$ median absolute deviation = median of the absolute differences between the $t t b(90)$ values and the calculated parameter, median(ttb(90)), the latter being the median of the $t t b(90)$ values $^{8}$.

Following the guideline suggested by Iglewiz and Hoaglin ${ }^{8}$, in this work $t t b(90)$ values with $Z$ (mod) values $>3.5$ are identified as outliers and not, therefore, used in the subsequent calculation of the average $t t b(90)$ value, $t t b(90)_{a v}$, and its standard deviation, $\sigma$. The results of this statistical analysis of the data in figure 3, i.e. the eight samples of commercial selfcleaning glass run by Cristal, are given in Table 1, which comprises values, for each of the samples, of: $R(\max ), R(\min ), R_{t}(90), t t b(90), Z(\bmod )$, as well as the decision whether the $t t b(90)$ value should be considered an outlier or not (answer: yes, if $Z(\bmod )$ is > 3.5). Other information given includes: a final value of the average of the non-outlier $t t b(90)$ values, $t t b(90)_{a v}$ and its standard deviation, $\sigma$ and the number of points, $n$, used.

Table 1: Results of analysis of $\mathrm{Rz}$ ink on commercial self-cleaning glass samples from Cristal

\begin{tabular}{|c|c|c|c|c|c|c|}
\hline Sample & $R(\max )$ & $R(\min )$ & $R_{t}(90)$ & $t t b(90) / \mathrm{s}$ & $Z(\bmod )$ & Outcome \\
\hline \#1 & 0.3991 & 0.1882 & 0.3780 & 195.5 & 0.14 & OK \\
\hline$\# 2$ & 0.4022 & 0.1854 & 0.3805 & 200.2 & 0.14 & OK \\
\hline \#3 & 0.3960 & 0.1988 & 0.3763 & 188.3 & 0.55 & OK \\
\hline$\# 4$ & 0.4023 & 0.1836 & 0.3804 & 201.7 & 0.22 & OK \\
\hline \#5 & 0.3944 & 0.1962 & 0.3746 & 184.0 & 0.80 & OK \\
\hline \#6 & 0.4060 & 0.1790 & 0.3833 & 225.9 & 1.62 & OK \\
\hline \#7 & 0.4001 & 0.1865 & 0.3787 & 298.6 & 5.83 & Outlier* \\
\hline \multirow[t]{4}{*}{$\# 8$} & 0.3910 & 0.2042 & 0.3723 & 158.1 & 2.30 & OK \\
\hline & & & & & $t t b(90)_{a v}$ & $193 \mathrm{~s}$ \\
\hline & & & & & $\sigma$ & 21 s (11\%) \\
\hline & & & & & $n$ & 7 \\
\hline
\end{tabular}

*: red print indicates outlier 
Similar tables were generated using the data from the digital images recorded by each of the four other participants in the RR test for the Rz ink on commercial self-cleaning glass trial and all the key results, are summarised in Table 2, which comprises, for each of the 5 groups, the $t t b(90)$ values for all the samples they ran and the associated $t t b(90)_{a v}$ and $\sigma$ values they calculated. A value for the repeatability standard deviation, $s_{r}$, was then calculated using the expression ${ }^{9}$ :

$$
S_{r}=\sqrt{\sum_{1}^{p} \sigma^{2} / p}
$$

where, $p=$ number of participating laboratories (5 in this case). And the reproducibility standard deviation, $s_{R}$, was then calculated using the expression ${ }^{9}$ :

$$
S_{R}=\sqrt{\left(s_{x}^{2}+\frac{s_{r}^{2}(n-1)}{n}\right)}
$$

where, $s_{x}$ is the standard deviation associated with the calculation of the average of the averages and $n$ is the number (usually $n=8$ ) of samples used to calculate the value of $s_{r}$. In addition, Table 2 contains the overall $t t b(90)$ value, $t t b(90)_{\mathrm{T}}$, which is the average of the $t t b(90)_{a v}$ values, and its standard deviation, $s_{x}$. A brief discussion of the repeatability and reproducibility exhibited by the inks tests for the 5 different materials tested here is given later.

The Rz ink test was also used to assess the activity of a photocatalytic paint of moderate activity and a typical set of $R_{t}$ vs $t$ profiles for an Rz ink on 8 samples of the paint on a glass substrate, as reported by ICT as part of their part of the RR trial, are illustrated in Figure 4. The overall results of the RR trial on this material are also summarised in table 2, and in the same format as that for the commercial self-cleaning glass. 
Table 2: Summaries of the results of the Rz ink RR trails on commercial self-cleaning glass and a medium activity, photocatalytic paint*.

\begin{tabular}{|c|c|c|c|c|c|c|c|c|c|c|c|}
\hline \multicolumn{12}{|c|}{ MEDIUM GLASS } \\
\hline & \multicolumn{8}{|c|}{ Test Results, $\mathrm{x}$} & \multirow[b]{2}{*}{ Average } & \multirow[b]{2}{*}{ S. D. } & \multirow[b]{2}{*}{ \% S. D. } \\
\hline & 1 & 2 & 3 & 4 & 5 & 6 & 7 & 8 & & & \\
\hline Aalborg & 228.6 & 182.7 & 237.0 & 264.0 & 256.2 & 182.4 & 225.8 & 230.6 & 225.91 & 29.95 & 13 \\
\hline Cristal & 195.5 & 200.2 & 188.3 & 201.7 & 184.0 & 225.9 & 298.6 & 158.1 & 193.39 & 20.56 & 11 \\
\hline DIT & 191.6 & 239.1 & 235.8 & 247.4 & 305.1 & 194.3 & 257.1 & 263.4 & 241.73 & 36.95 & 15 \\
\hline ICT & 232.3 & 217.2 & 195.9 & 224.5 & 239.2 & 222.1 & 258.3 & 227.5 & 227.13 & 17.93 & 8 \\
\hline \multirow[t]{5}{*}{ QUB } & 178.6 & 246.4 & 237.9 & 244.2 & 278.3 & 228.6 & 259.9 & 276.7 & 243.83 & 31.75 & 13 \\
\hline & \multicolumn{8}{|c|}{ Average of Averages } & 226.39 & & \\
\hline & \multicolumn{8}{|c|}{ SD of averages } & 20.18 & $(9 \%)$ & \\
\hline & \multicolumn{8}{|c|}{ Repeatability SD } & 28.34 & $(13 \%)$ & \\
\hline & \multicolumn{8}{|c|}{ Reproducibility SD } & 33.32 & $(15 \%)$ & \\
\hline \multicolumn{12}{|c|}{ MEDIUM PAINT } \\
\hline & \multicolumn{8}{|c|}{ Test Results, $\mathrm{x}$} & & & \\
\hline & 1 & 2 & 3 & 4 & 5 & 6 & 7 & 8 & Average & S. D. & \% S. D. \\
\hline Aalborg & 416.5 & 418.8 & 409.4 & 432.3 & 375.1 & 423.9 & 383.7 & 383.8 & 405.44 & 21.51 & 5 \\
\hline Cristal & 316 & 326.5 & 327.4 & 340.4 & 293.1 & 275.5 & 317.6 & 344.5 & 317.63 & 23.28 & 7 \\
\hline DIT & 446.5 & 481.1 & 465.1 & 456.2 & 477.6 & 474.3 & 503 & 451.4 & 469.40 & 18.51 & 4 \\
\hline ICT & 336.1 & 308.7 & 359.8 & 359.6 & 355.6 & 376.8 & 356.5 & 377.2 & 353.79 & 22.37 & 6 \\
\hline \multirow[t]{5}{*}{ QUB } & 281.1 & 296.1 & 333.1 & 320.3 & 290.2 & 285.2 & 287.8 & 289.4 & 288.30 & 5.05 & 2 \\
\hline & \multicolumn{8}{|c|}{ Average of averages } & 366.91 & & \\
\hline & \multicolumn{8}{|c|}{ SD of averages } & 72.06 & $(20 \%)$ & \\
\hline & \multicolumn{8}{|c|}{ Repeatability SD } & 19.36 & $(5 \%)$ & \\
\hline & \multicolumn{8}{|c|}{ Reproducibility SD } & 74.30 & $(20 \%)$ & \\
\hline
\end{tabular}

*: red print indicates outliers 


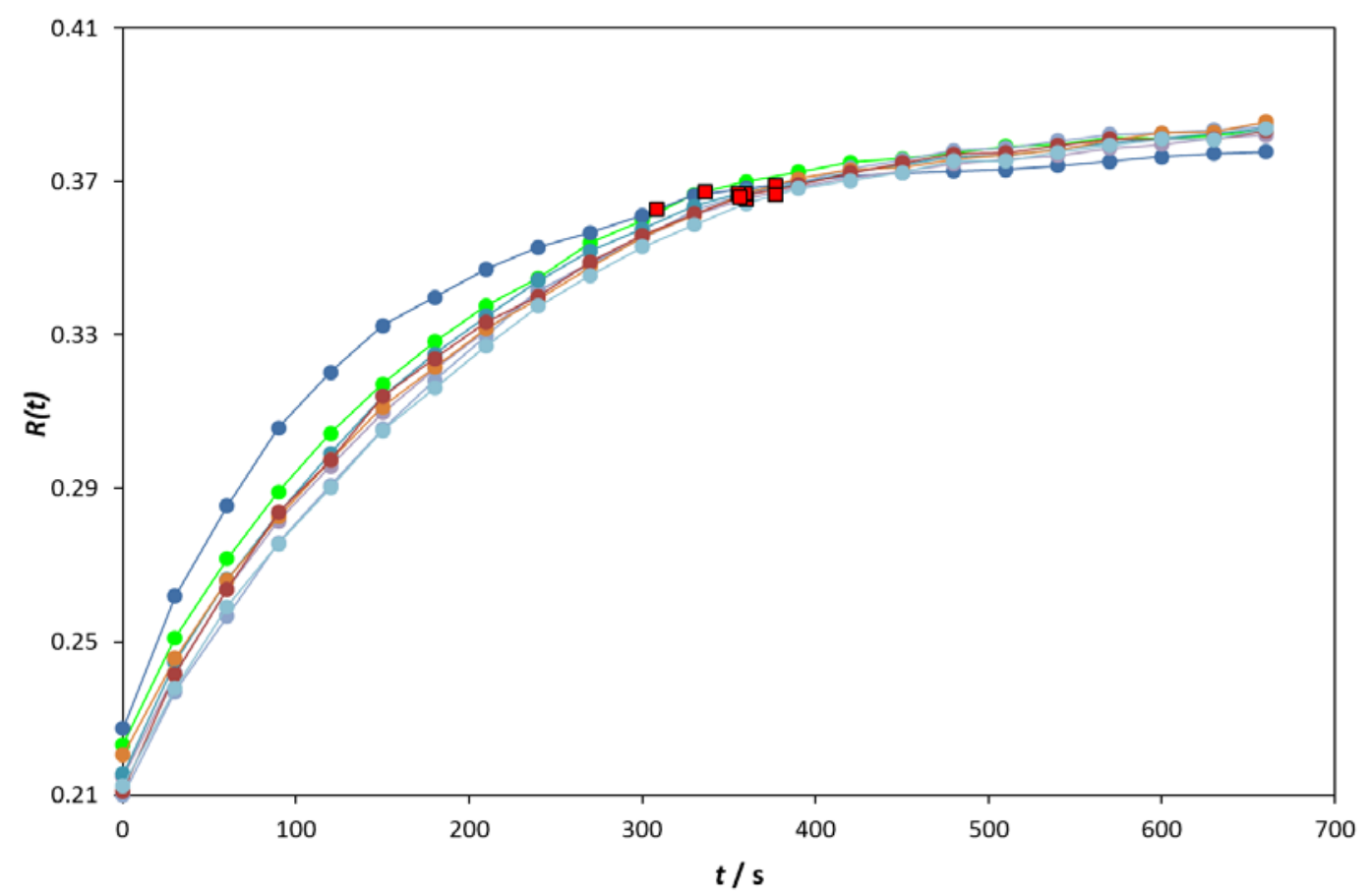

Figure 4: Plots of $R_{t}$ vs $t$ profiles for an Rz ink on 8 samples of medium activity paint as reported by ICT as part of the RR tests. The solid red box points identify the calculated $t t b(90)$ values for each of the curves.

\section{The AV7 ink test}

The redox azo dye, AV7, which changes from pink to colourless, was found to be more difficult to reduce than $\mathrm{Rz}$ and so was considered to be a likely, appropriate alternative to $\mathrm{Rz}$ for assessing the efficacies of high (rather than medium) activity photocatalytic materials, such as commercial photocatalytic paint and titania solgel coated glass, for which the $\mathrm{Rz}$ ink is inappropriate as it is too readily photo-reduced by these materials. One reason the sol-gel coated glass is much more active than commercial self-cleaning glass is due to its greater UVA-absorbing nature, due in turn to its greater thickness, i.e. $70 \mathrm{~nm}$ compared to $15 \mathrm{~nm}$. In the AV7 ink, the red dye is bleached by the photocatalytic reductive process summarised by eqns (1) - (3), and the key RGB colour component to change as a consequence is green. Thus, using this ink, the key parameter to plot in order to derive a value for $t t b(90)$ is $R_{t}$ vs $t$, where the latter is defined by eqn (5). Figures 5 and 6 are plots of typical sets of $R_{t}$ vs $t$ profiles for an AV7 ink on 8 samples of the highly active commercial photocatalytic paint and titania sol-gel coated glass samples, respectively. The overall results of the RR trials using this ink on these two materials are summarised in Table 3. 


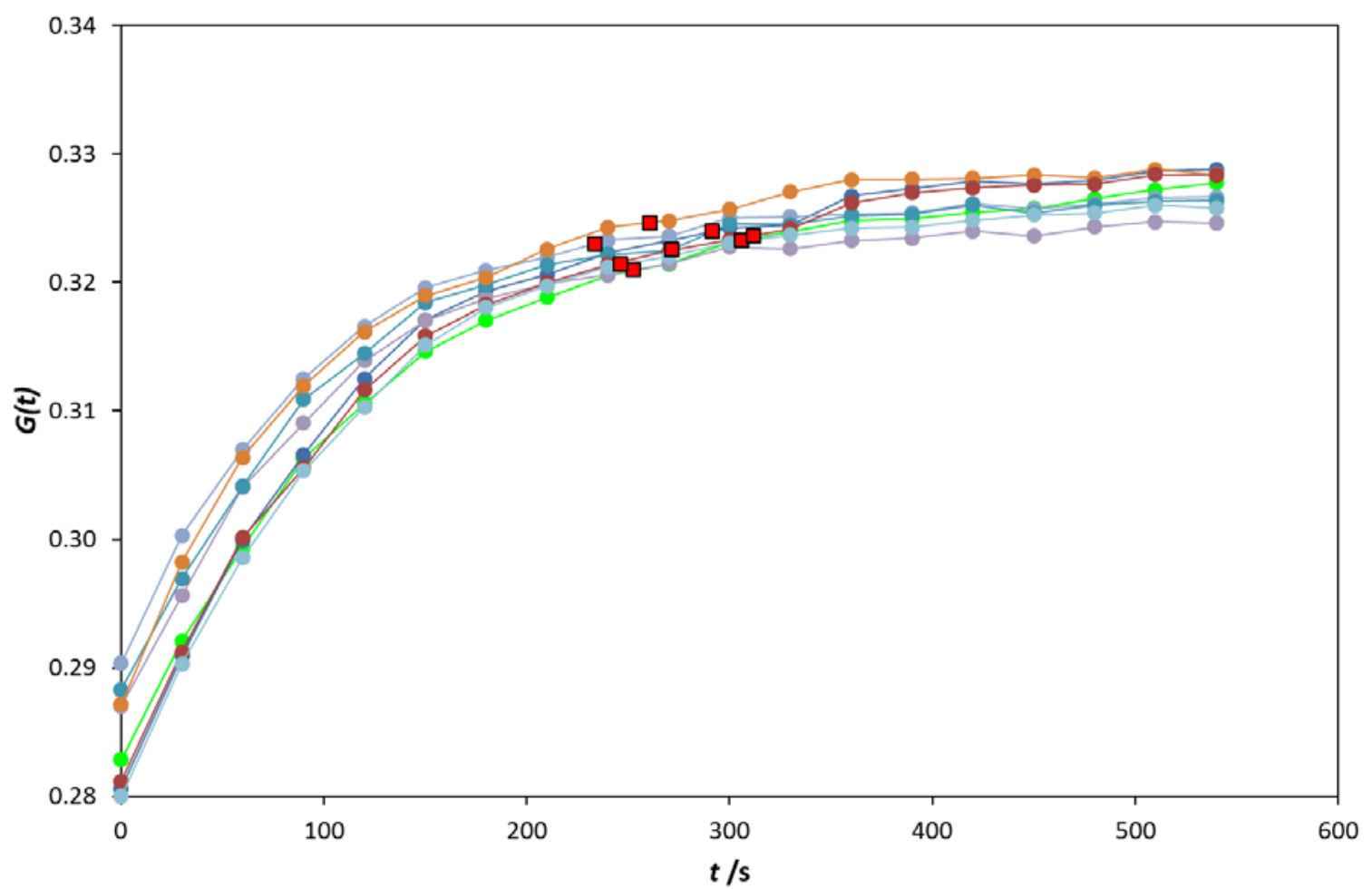

Figure 5: Plots of $R_{t}$ vs $t$ profiles for an AV7 ink on 8 samples of high activity commercial paint as reported by ICT as part of the RR tests.

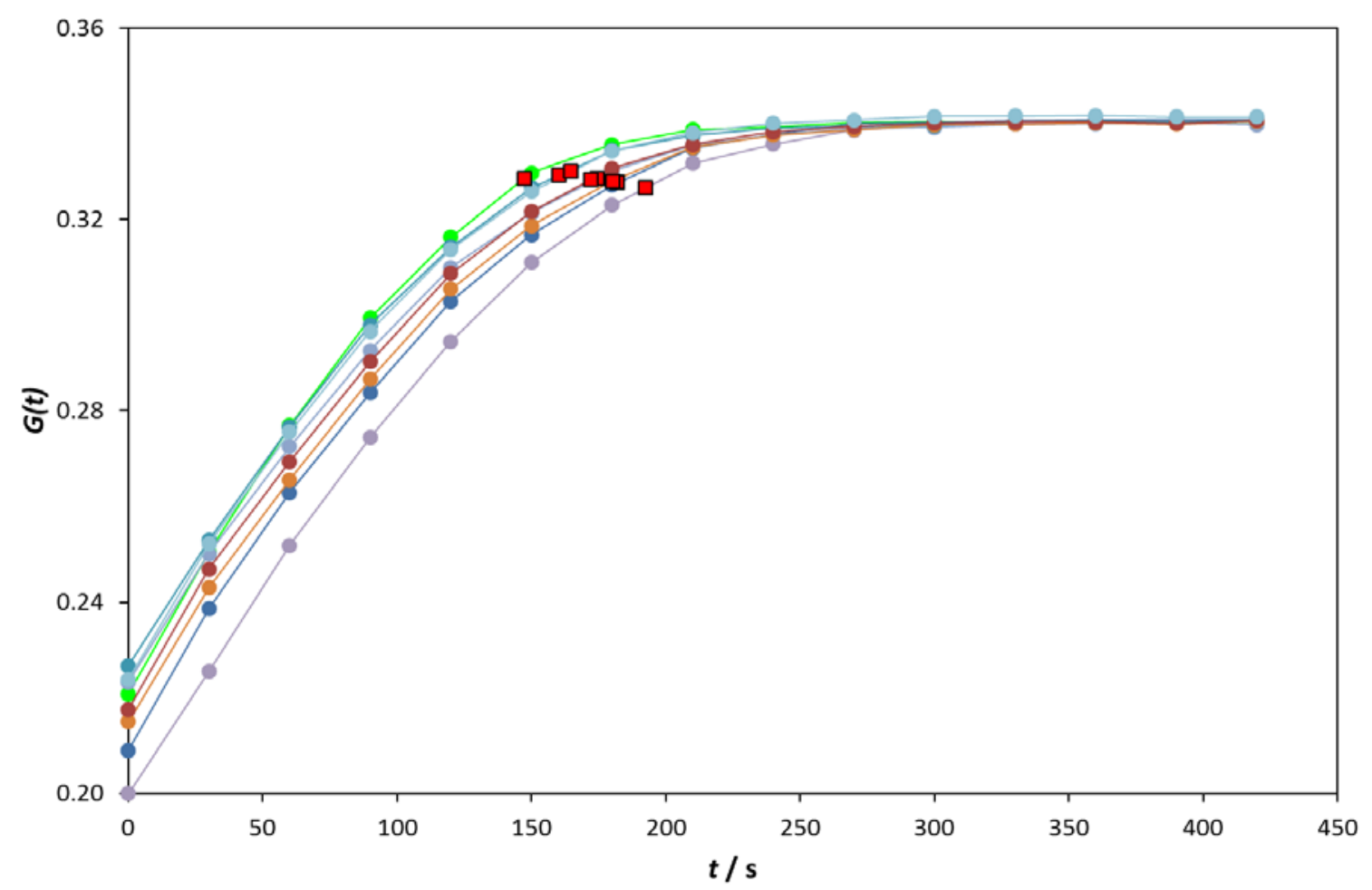

Figure 6: Plots of $R_{t}$ vs $t$ profiles for an AV7 ink on 8 samples of high activity titania sol-gel coated glass as reported by Aalborg as part of the RR tests. 
Table 3: Summaries of the results of the AV7 ink RR trails on high activity (fast), commercial photocatalytic paint and titania sol-gel coated glass.

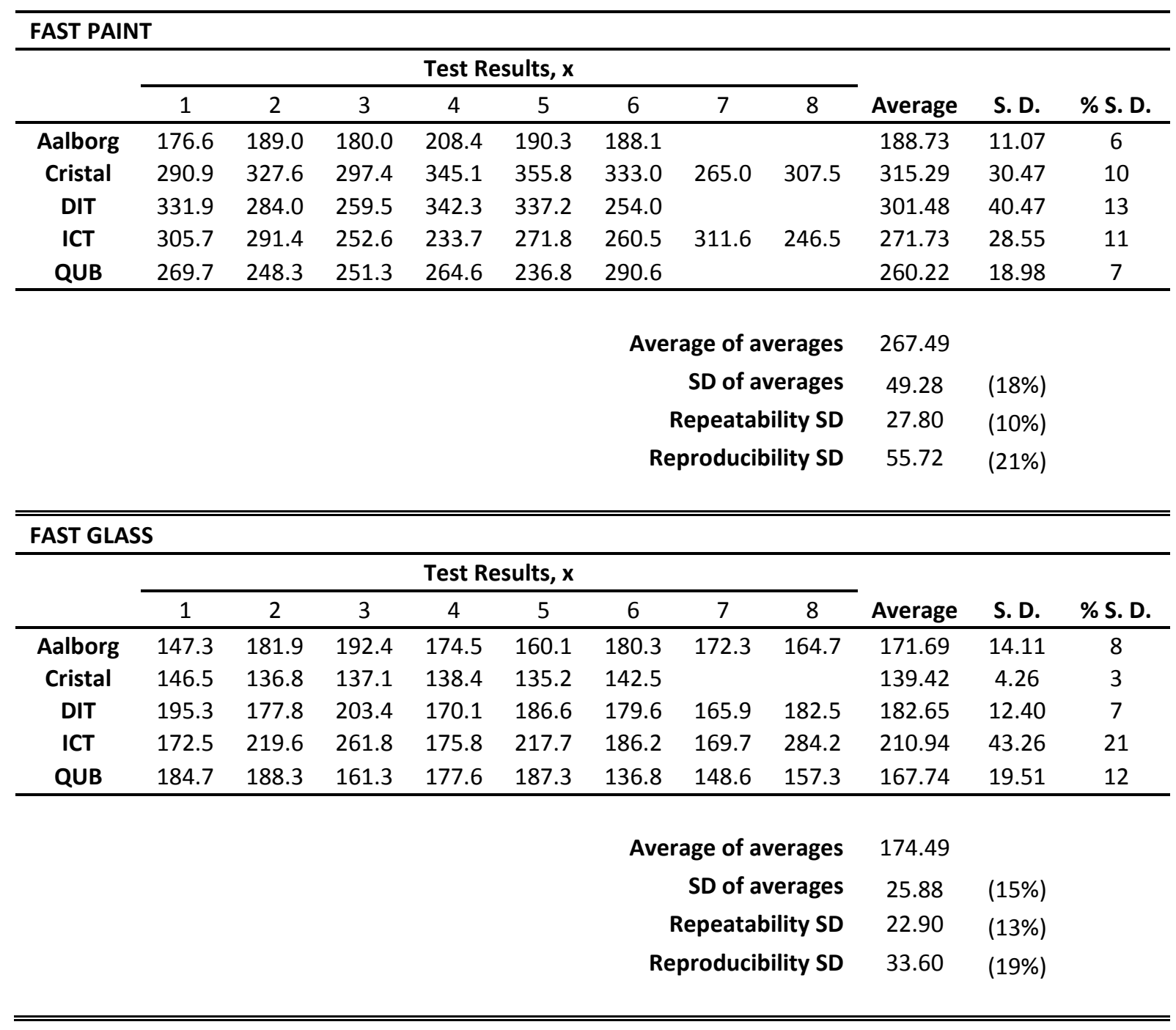

The BB66 ink test

The azo dye, BB66, which changes from blue to colourless, was found to be much easier to reduce than $\mathrm{Rz}$ and so appeared to be an appropriate alternative to $\mathrm{Rz}$ for assessing the efficacies of photocatalytic materials which exhibit activities that are much lower than that of most examples of commercial self-cleaning commercial glass. Examples of such low activity materials include: commercial photocatalytic tiles, which utilised the patented Hydrotect technology of $\mathrm{TOTO}^{10}$. Initial work showed that the commercial photocatalytic tiles used here were not able to change the colour of an Rz ink (blue to pink) in $<45 \mathrm{~min}$, but would change the colour (blue to colourless) of a BB66 ink within 8 min. As with the Rz ink, red is the key colour component of the RGB analysed image which changes most significantly 
when a BB66 (blue) is bleached photocatalytically via reactions (1) - (3), and thus the profile of $R_{t}$ vs. $t$ for each sample tested using this ink was calculated using eqn (4) and the data so generated were then used to determine the $t b(90)$ value for the sample under test. Figure 7 illustrates a typical set of $R_{t}$ vs $t$ profiles for a BB66 ink on 8 samples of the low activity commercial photocatalytic tiles used in this work, as reported by QUB, and the overall results of the RR trials using this ink on this material are summarised in Table 4.

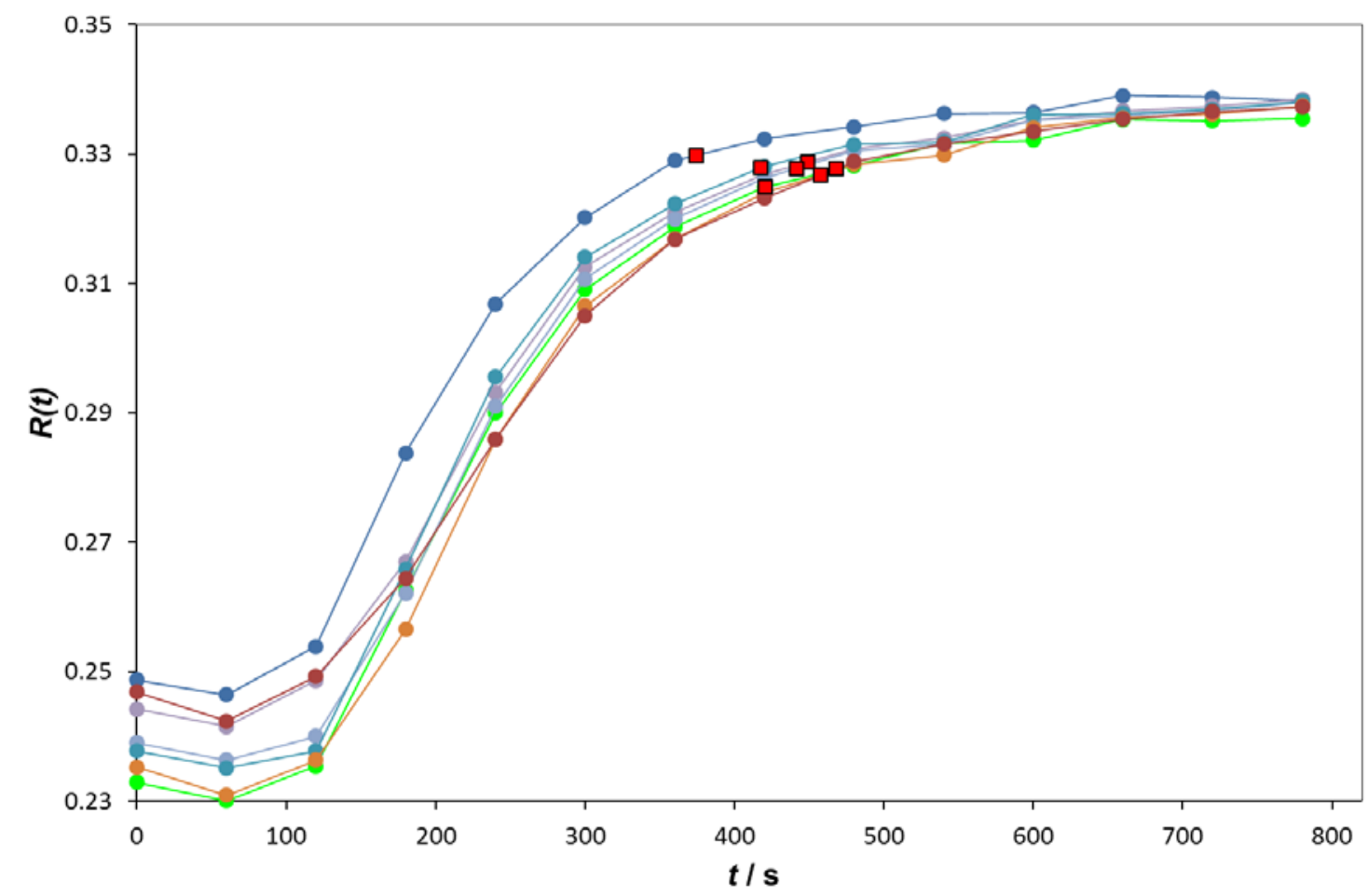

Figure 7: Plots of $R_{t}$ vs $t$ profiles for a BB676 ink on 8 samples of low activity commercial tiles as reported by QUB as part of the RR tests. 
Table 4: Summary of the results of the BB66 ink RR trials on low activity, commercial photocatalytic tiles*.

\begin{tabular}{|c|c|c|c|c|c|c|c|c|c|c|c|}
\hline \multicolumn{12}{|c|}{ SLOW TILES } \\
\hline & \multicolumn{8}{|c|}{ Test Results, $\mathrm{x}$} & \multirow[b]{2}{*}{ Average } & \multirow[b]{2}{*}{ S. D. } & \multirow[b]{2}{*}{ \% S. D. } \\
\hline & 1 & 2 & 3 & 4 & 5 & 6 & 7 & 8 & & & \\
\hline Aalborg & 282.1 & 298.0 & 390.5 & 465.8 & 304.1 & 359.5 & 307.9 & 357.0 & 345.61 & 61.21 & 18 \\
\hline Cristal & 637.3 & 1079.9 & 652.6 & 711.9 & 658.1 & 635.4 & 603.7 & 1067.4 & 649.83 & 35.84 & 6 \\
\hline DIT & 412.3 & 477.3 & 457.2 & 455.3 & 416.4 & 433.0 & & & 441.92 & 25.58 & 6 \\
\hline ICT & 316.1 & 314.8 & 594.1 & 346.1 & 446.1 & 391.4 & 358.4 & 621.6 & 423.58 & 121.58 & 29 \\
\hline \multirow[t]{5}{*}{ QUB } & 420.5 & 374.1 & 448.8 & 441.7 & 417.8 & 457.8 & 468.4 & & 432.73 & 31.75 & 7 \\
\hline & \multicolumn{8}{|c|}{ Average of averages } & 458.73 & & \\
\hline & \multicolumn{8}{|c|}{ SD of averages } & 113.48 & $(25 \%)$ & \\
\hline & \multicolumn{8}{|c|}{ Repeatability SD } & 65.54 & $(14 \%)$ & \\
\hline & \multicolumn{8}{|c|}{ Reproducibility SD } & 128.98 & $(28 \%)$ & \\
\hline
\end{tabular}

*: red print indicates outliers

\section{Repeatability and Reproducibility}

Repeatability is the degree of agreement of tests or measurements on replicate specimens by the same observer in the same laboratory, i.e. repeatability is a measure of how well the same experimenter can generate the same results. In contrast, reproducibility is the ability of an entire experiment to be reproduced by someone, other than the original experimenter, working independently. Measures of method repeatability and reproducibility are usually provided via a statistical analysis of the results arising from the method. For each material tested in the RR trials, the percentage standard deviations associated with the repeatability and reproducibility of the ink test used were calculated and the results of this work are reported in tables 3- 4 and illustrated in Figure 8. 


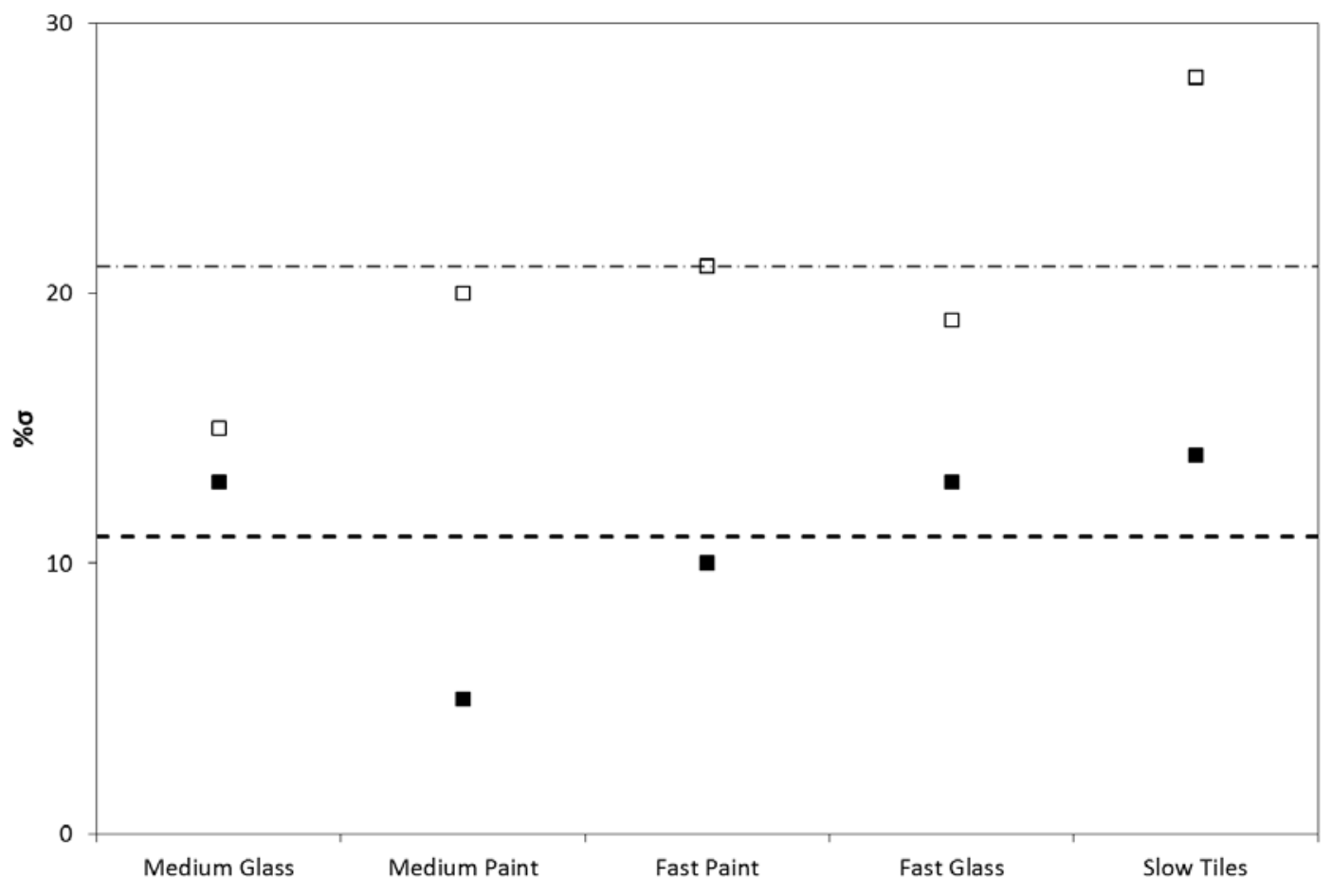

Figure 8: Calculated \%standard deviations of repeatability ( $\boldsymbol{\square}$ ) and reproducibility ( $\square$ ) for the 5 materials tested using the photocatalytic activity indicator inks based on Rz (medium activity), AV7 (high activity) and BB66 (low activity).

From the results of this work it would appear that the repeatability of the inks (for measuring activity) is typically ca. $11 \%$ and the reproducibility ca. $21 \%$, both shown as broken lines in figure 8. Although the values are not insignificant, they are also not too discouraging with respect to using the inks as an assessment method, especially when put in the context of the values reported for other photocatalyst tests. For example, in the methylene blue test, ISO 10178:2010 ${ }^{11}$, for self-cleaning glass alone, the reported repeatability and reproducibility \%standard deviations were: $9 \%$ and 31\% respectively, compared to 13 and 15\%, respectively, in this work for the same photocatalytic material type. The point also shouldn't be lost here that, as is apparent from the results in figures 3-7, the photocatalyst activity ink tests are, unlike all their current ISO counterpart tests for photocatalytic activity, able to probe the photocatalytic activities of a wide range materials with very different activities AND often in less than $10 \mathrm{~min}$, whereas most ISO tests require an irradiation period of 3-5 h. 


\section{Methods - other than digital photography - for assessing ink colour change}

In this paper the colour changes exhibited by the different inks, (blue to pink (Rz), blue to colourless (BB66) and pink to colourless (AV7), which were used to assess the activities of the five different photocatalytic materials by the five different groups have been monitored via their colour digital images and the RGB colour model. In particular, the monitoring, as a function of $t$, of the normalised red component, $R_{t}$, as calculated using eqn (4), for the blue inks, Rz and BB66, and the normalised green component, $R_{t}$, as calculated using eqn (5), for the red (AV7) ink. However, there are obvious, alternative - albeit just single sample methods for monitoring colour change include UV/Vis absorption spectroscopy and Lab colour space, as measured using diffuse reflectance spectroscopy. This prompts the question: is, for any sample, the $t t b(90)$ value measured using either one of these other methods the same as that measured using RGB colour analysis and digital photography?

Thus, in one set of experiments, an Rz ink coated sample of self-cleaning glass was irradiated with UVA light and the photocatalytic induced colour change monitored by UV/Vis absorption spectroscopy, digital photography and diffuse reflectance spectroscopy, to

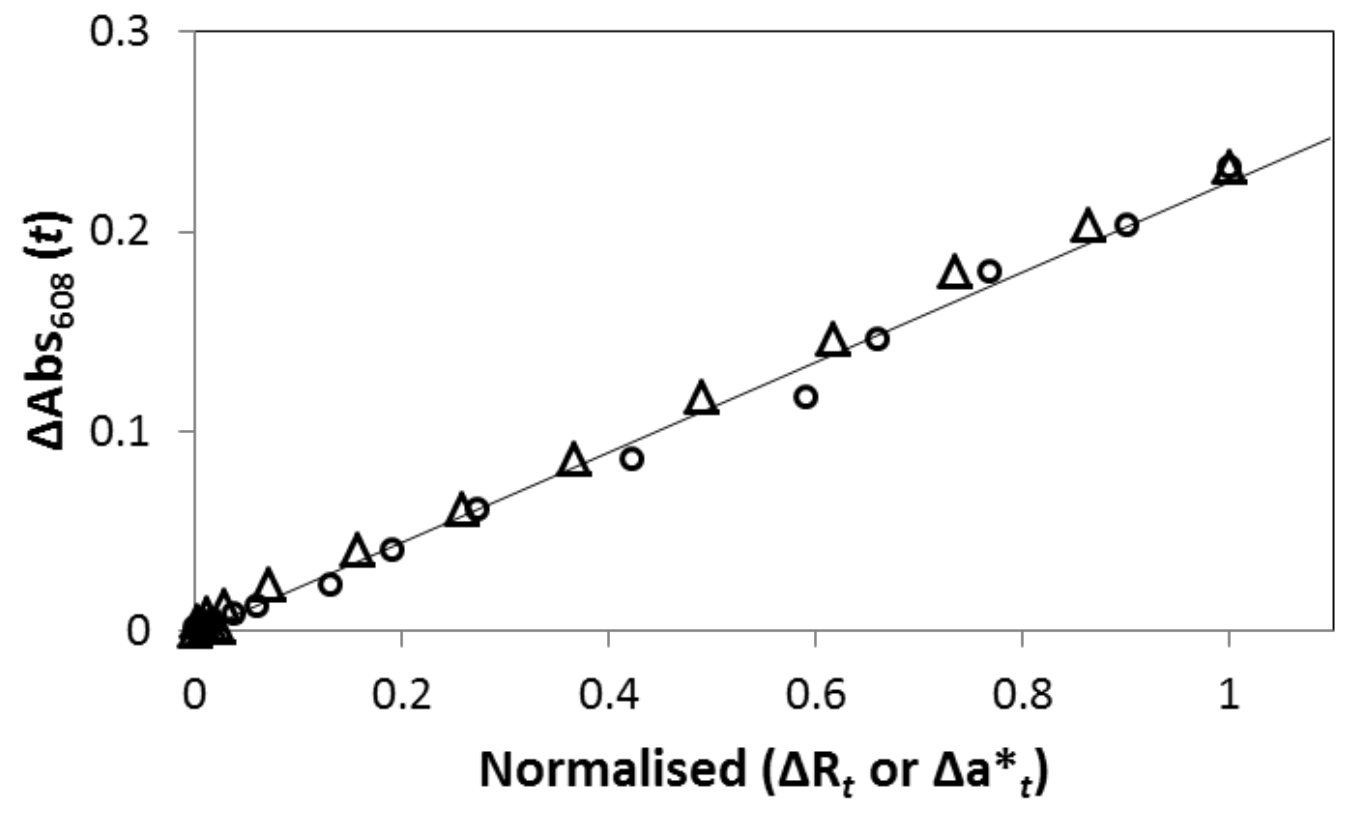

Figure 9: Plot of $\Delta \mathrm{Abs}_{608}(t)$ vs normalised values of $\Delta R_{t}(O)$ and $\Delta a_{t}(\triangle)$ as measured using RGB and Lab colour analysis, for a sample of self-cleaning glass, coated with an Rz ink and subsequent irradiated with UVA light $\left(2 \mathrm{~mW} \mathrm{~cm}^{-2}\right)$. 
generate values of $\Delta \mathrm{Abs}_{608}(t), \Delta R_{t}$ and $\Delta a_{t}$ values for each irradiation time, $t$. Figure 9 is a plot of the data so obtained in the form of $\Delta \mathrm{Abs}_{608}(t)$ vs the corresponding values of: $\Delta R_{t}$ and $\Delta a_{t}$ (normalised so that they can be plotted on the same graph). The results of this show that both $\Delta R_{t}$ and $\Delta a_{t}$ are proportional to $\Delta \mathrm{Abs}_{608}(t)$, and, thus, all three techniques will generate the same $t t b(90)$ value for the same sample. In addition, since the rate of change of $\Delta \mathrm{Abs}_{608}(t)$ is proportional to the photocatalytic activity of the sample, as measured by the destruction of stearic acid for example, then so too are the rates of change of $\Delta R_{t}$ and $\Delta a_{t}$, an inverse measure of which is the value of $t t b(90)$, proportional to the photocatalytic activity of the sample. Thus, any of the major colour-measuring techniques, i.e. UV/Vis absorption spectroscopy (transparent samples only), digital photography or diffuse reflectance spectroscopy, can be used, in conjunction with an appropriate photocatalyst activity indicator ink, to measure the activity of a photocatalytic film, via its value of $t t b(90)$. However, digital photography, unlike the other two methods, has the added benefit of simultaneous sampling of many samples, or mapping of activity over a large sample, as shown by others ${ }^{12,13}$.

\section{Conclusion}

Three photocatalyst inks based on the redox dyes, Rz, AV7 and BB66 can be used to assess the photocatalytic activities of a variety of different materials, such as commercial paint, tiles and glass and laboratory made samples of sol-gel coated glass and paint, which collectively exhibit a wide range of activities that cannot currently be probed by only one of the existing ISO tests. The ink tests are fast (typically $<10 \mathrm{~min}$ ), simple to employ and inexpensive. Previous work suggests the ink tests correlate linearly with other photocatalytic tests such as the photomineralisation of stearic acid. In a series of round robin tests, their average repeatability and reproducibility are ca. $11 \%$ and ca $21 \%$, respectively, which compare well with those reported for the current ISO tests. This approach to the assessment of the activities of photocatalyst films has the potential to improve significantly the degree of quality control exercised on commercial photocatalyst products, and improve the abilities of researchers to screen rapidly the activities of new materials, especially when used with combinatorial synthesis methods and activity mapping ${ }^{12,13}$.

\section{Acknowledgements}

The authors thank the EU (FP7 NMP-2012-CSA-6 INTEC) for helping to fund the round robin tests. 


\section{References}

1. A. Mills, C. Hill and P. K. J. Robertson, J. Photochem. Photobiol. A: Chem., 2012, 237, 7-23.

2. A. Mills, Appld. Catal. B: Environmental, 2012, 128, 144-149.

3. A. Mills, J. Hepburn, D. Hazafy, C. O’Rourke, J. Krysa,M. Baudys, M. Zlamal, H. Bartkova, C. E. Hill, K. R. Winn, M. E. Simonsen, E. G. Søgaard, S. C. Pillai, N. S. Leyland, R. Fagan, F. Neumann, C. Lampe and T. Graumann , J. Photochem. Photobiol. A; Chem., 2013, 272, 18-20.

4. A. Mills, J. Wang, S.-K. Lee and M. Simonsen, Chem. Commun., 2005, 2721-2723.

5. J. Zita, J. Krysa, A. Mills, J. Photochem. Photobiol. A 203 (2009) 119-124.

6. http://rsb.info.nih.gov/ij/download.html; accessed April 2014

7. A. Mills, C. O’Rourke, K. Lawrie, and S. Elouali, ACS Appl. Mater. Interfaces, 2014, 6 (1), pp 545-552

8. B. Iglewicz and D. Hoaglin, in The ASQC Basic References in Quality Control: Statistical Techniques, E. F. Mykytka (ed.), Amer Society for Quality, Wisconsin, 16, 1993.

9. ASTM E691-13, 'Standard practice for conducting an interlaboratory study to determine the precision of a test', ASTNM International Conshohoken, PA, 2013, DOI: 10.1520/E0691-13

10. http://www.toto.co.jp/hydrotect/lang.html; accessed April 2014.

11. ISO 10678: 2010, 'Fine ceramics, advanced technical ceramics) Determination of photocatalytic activity of surfaces in an aqueous medium by degradation of methylene blue', ISO, Geneva, 2010.

12. A. Mills, N. Wells, C. O’Rourke, Catal. Today 230 (2014) 245-249.

13. A. Kafizas, A., C. Crick, and I.P. Parkin, J. Photochem. Photobiol. A: Chem, 2010, 216, 156-166.

14. A. Kafizas and I.P. Parkin, J. Mater. Chem., 2010, 20, 2157-2169.

Cite this as Mills et al Journal of Photochemistry and Photobiology A: Chemistry 290 (2014) $63-71$

Article link: http://www.sciencedirect.com/science/article/pii/S1010603014002597 